\title{
LA CUENTA DE INVERSIÓN UNA HERRAMIENTA PARA EVALUAR LOS OBJETIVOS GENERALES PLANTEADOS EN LOS PROGRAMAS DE GOBIERNO. EL CASO DE LA PROVINCIA DE SANTA FE 2004-2015
}

\author{
Tesista \\ Leandro Dionisio Yossen \\ Directora de tesis \\ María Cristina Gonnet
}

La sociedad argentina demanda, actualmente, una especie de balance respecto de la gestión pública llevada adelante por el Poder Administrador. Recordemos, que a partir del 10 de diciembre de 1983 la República Argentina comenzó un nuevo camino democrático, el que prosigue ininterrumpidamente hasta nuestros días. En este contexto, más de treinta y cinco años de gobiernos constitucionales han provocado cambios en los requerimientos sociales, que además de los ya tradicionales sobre provisión y/o acceso a bienes y servicios, regulación, seguridad, etc., suma la de exigir o reclamar la evaluación de las propuestas generales de gobierno planteadas en su momento a la sociedad. En virtud de ello, el presente trabajo expone una metodología que nos permite evaluar a través de los contenidos de las Cuentas de Inversión, en especial sus clasificaciones presupuestarias o financieras, a algunos de los objetivos generales propuestos a la sociedad en los Programas de Gobierno. Puntualmente, se analizan los Programas de
Gobierno presentados por el Frente Progresista Cívico y Social en los años 2007 y 2011 en el ámbito de la Provincia de Santa Fe.

El aporte de esta investigación radica en que expone una metodología que nos permite confrontar el Programa de Gobierno con la Acción de Gobierno efectivamente llevada a cabo, en base al contenido de las Cuentas de Inversión de la Provincia de Santa Fe. La elección de esta última herramienta se sustenta en que es el único instrumento disponible que puede reflejar lo realizado, lo concretado por la gestión de gobierno en forma integrada; en cambio el presupuesto, aunque también integrador, hace mención a la previsión a la intención de realizar tal o cual política pública. En ese mismo sentido, la doctrina señala a la Cuenta de Inversión como la herramienta capaz de evaluar las prioridades y el rumbo de un gobierno cuando se procede a su examen anual; agregando, que la sumatoria de estos análisis a lo largo del tiempo deja entrever el accionar gubernamental de todo el período seleccionado. La metodología 
de este trabajo se encuentra básicamente alineada a estas apreciaciones. Por otro lado, la normativa vigente en la República Argentina y sus Provincias establece que la Cuenta de Inversión o la Cuenta General del Ejercicio es el instrumento sobre el cual el Poder Legislativo efectiviza el control de la gestión realizada por el Poder Ejecutivo.

Otra particularidad que se ha tenido en cuenta es la demanda de la sociedad respecto a que la información que se produzca sea objetiva, fehaciente y técnicamente comprensible para poder ser interpretada en un marco de confiabilidad. En la actualidad, se encuentran publicadas en el portal web oficial de la Provincia de Santa Fe las Cuentas de Inversión pertenecientes a los ejercicios 2003 en adelante; y aunque resumen la ejecución presupuestaria, exponen una cantidad significativa de datos que requieren de un importante esfuerzo para poder ser transformados en información. La sociedad, en general, no cuenta con los recursos y conocimientos técnicos necesarios para realizarlos.

Por ello, este trabajo puso a prueba a las variables educación y cultura, infraestructura y descentralización. Estas han sido, en primer lugar, elegidas de los Programas de Gobierno sobre los cuales se basaron las campañas electorales del Frente Progresista Cívico y Social de la Provincia de Santa Fe en los años 2007 y 2011. Y, en segundo lugar, por la importancia que estas tienen para el desarrollo de un Estado contemporáneo.

Para dar respuesta a esta problemática, en general, la estructura metodológica propone primeramente vincular a un determinado objetivo general de gobierno con algún contenido de la Cuenta de Inversión que explique, de forma general, lo ocurrido. En segundo lugar, utiliza una serie de indicadores que permiten obtener la participación porcentual anual de los gastos (porcentaje), la participación porcentual promedio de los gastos en cada período (razón o promedio) y el cambio de participación promedio de los gastos de un período a otro (tasa de variación). Estos indicadores se aplican sobre el total del gasto de la Administración Provincial de la Provincia de Santa Fe entre los años 2004 a 2015; el período 2004-2007 (base) y los períodos analizados 2008-2011 y 2012-2015. Concretamente, para el tratamiento de los datos financieros, se construyeron matrices anuales que especifican el porcentaje de participación relativa de la variable analizada en relación al total del gasto de la Administración Provincial; a su vez, estas se consolidan en una matriz que contiene la totalidad de los años de cada período.

Esta metodología, así planteada, nos permite comparar al ciclo de gobierno bajo estudio contra el establecido como base; obteniéndose como resultado la cuantía y el sentido de su cambio en el tiempo. En los casos en que la evidencia sea válida y suficiente la información conseguida nos permitirá inferir si el Poder Ejecutivo ha destinado una mayor cantidad relativa de fondos al cumplimiento del objetivo general propuesto en el programa de gobierno o, en su defecto, si se ha mantenido o disminuido; presumiendo, en estos últimos casos, que será de difícil concreción la mejora del objetivo general planteado. Céteris páribus la eficiencia del gasto del Estado entre los períodos analizados y el tomado como base.

Al procesar la información a través de la escala de medición (output posible: alta tendencia hacia el cumplimiento del objetivo general, moderada tendencia hacia el cumplimiento del objetivo general, leve tendencia hacia el cumplimiento del objetivo general, técnicamente neutra y no cumple) se obtuvieron los siguientes resultados:

- Variable educación y cultura: en ambos períodos analizados (2008-2011 y 20122015) la clasificación por finalidad/función 
obtuvo la calificación de "alta tendencia hacia el cumplimiento del objetivo general». Por lo que se infirió que el Ejecutivo Provincial fue en el sentido correcto para el logro de este objetivo general.

- Variable infraestructura: la clasificación económica del gasto arrojo el resultado, en ambos períodos, de "no cumple». Por lo que se infirió que no se fue en el sentido apropiado para el logro de este objetivo general. - Variable descentralización: en este caso se llegó a la conclusión de que, en su conjunto, no fue posible reunir elementos de juicios válidos y suficientes que nos permitan evaluar a la misma. Se observaron limitaciones tanto en el alcance como en la confiabilidad de la clasificación geográfica del gasto.

Los resultados obtenidos demuestran que la metodología desarrollada brinda información que nos permite realizar evaluaciones sencillas sobre los objetivos generales analizados. Por consecuencia de ello, se dota a la sociedad en general y a los demás estamentos sociales con una herramienta que facilita la realización de lo que podríamos llamar una especie de balance respecto de la gestión del
Poder Administrador. Cabe mencionar, que las limitaciones que se han observado se deben a la falta de contenidos, en las Cuentas de Inversión, que expliquen al objetivo general bajo estudio en forma consistente, como fue el caso de la variable descentralización. Es por ello, que se hace necesario avanzar aún más en los contenidos de las Cuentas de Inversión; tanto, desde un punto de vista financiero y sobre el manejo de variables reales medidas en unidades físicas. Además, la combinación de contenidos financieros y reales será lo que nos abrirá la puerta para poder tener una aproximación de la eficiencia del gasto del Estado.

Finalmente, se remarca que este trabajo abre nuevas preguntas relacionadas a la evolución que pudieron haber tenido otros objetivos generales, en el marco de la agenda política impulsada por el gobierno de la Provincia de Santa Fe en los períodos 2008-2011 y 2012-2015, como podrían ser las variables de salud, justicia y seguridad; $y$, a las respuestas que podría brindar esta metodología si se aplicara, con adecuaciones, en otras jurisdicciones provinciales o a nivel nacional. No obstante, estas cuestiones encontraran respuesta al ser objeto de estudio en otras investigaciones.

\section{PARA CITAR ESTE ARTÍCULO:}

Yossen, L.D. (2019). «Recensión de tesis: “La cuenta de inversión una herramienta para evaluar los objetivos generales planteados en los programas de gobierno. El caso de la provincia de Santa Fe 2004 2015"», DAAPGE, año 19, No 33 (jul-dic), 2019, pp. 165-167. Santa Fe, Argentina: UNL. 